Document downloaded from:

http://hdl.handle.net/10251/99664

This paper must be cited as:

Martínez Molada, E.; Singh, S.; Hueso Pagoaga, JL.; Gupta, D. (2016). Local convergence of a family of iterative methods for Hammerstein equations. Journal of Mathematical Chemistry. 54(7):1370-1386. doi:10.1007/s10910-016-0602-2

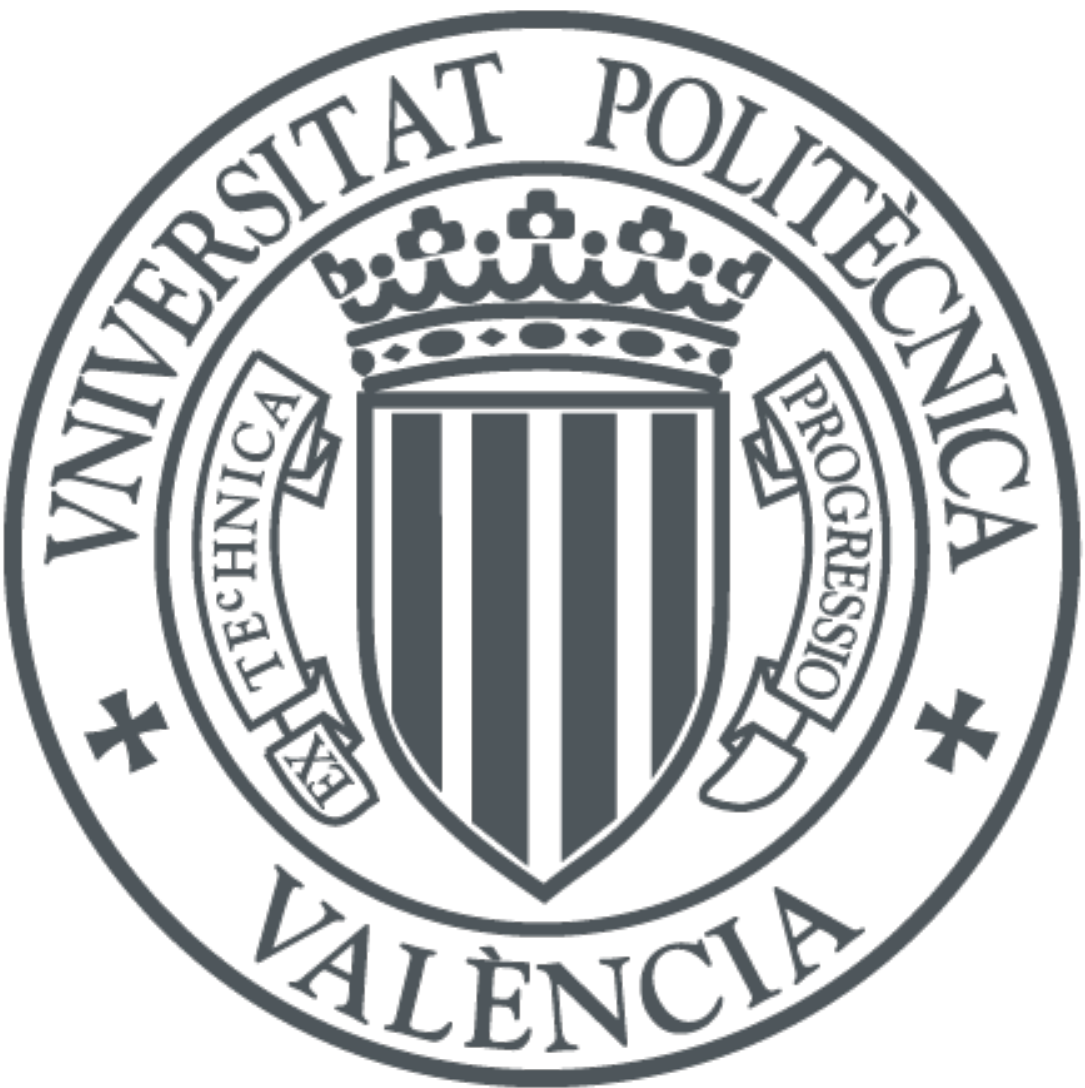

The final publication is available at

http://doi.org/10.1007/s10910-016-0602-2

Copyright Springer-Verlag

Additional Information 


\title{
Local convergence of a family of iterative methods for Hammerstein equations, CMMSE-15*
}

\author{
Eulalia Martínez ${ }^{(a) \dagger}$ Sukhjit Singh ${ }^{(b)}$, José L. Hueso ${ }^{(c)}$, Dharmendra K. Gupta ${ }^{(b)}$ \\ ${ }^{(a)}$ Instituto Universitario de Matemática Multidisciplinar. Universitat Politècnica de València \\ ${ }^{(b)}$ Department of Mathematics. Indian Institute of Technology Kharagpur \\ ${ }^{(c)}$ Instituto Universitario de Matemática Pura y Aplicada. Universitat Politècnica de València
}

\begin{abstract}
In this paper we give a local convergence result for a uniparametric family of iterative methods for nonlinear equations in Banach spaces. We assume boundedness conditions involving only the first Fréchet derivative, instead of using boundedness conditions for high order derivatives as it is usual in studies of semilocal convergence, which is a drawback for solving some practical problems. The existence and uniqueness theorem that establishes the convergence balls of these methods is obtained. We apply this theory to different examples, including a nonlinear Hammerstein equation that have many applications in chemistry and appears in problems of electro-magnetic fluid dynamics or in the kinetic theory of gases. With these examples we illustrate the advantages of these results. The global convergence of the method is addressed by analysing the behaviour of the methods on complex polynomials of second degree.
\end{abstract}

Keywords: Nonlinear systems Iterative method Banach space Local convergence Complex dynamics Hammerstein equation

2000 Mathematics Subject Classification: 47H99, $65 \mathrm{H} 10$.

\section{Introduction}

Solving nonlinear equations is an important branch of Numerical Analysis. A great variety of problems in sciences and engineering can be modeled by ordinary differential equations, partial derivative equations, integral equations, etc. [1]. After applying the corresponding

*This research was supported by Ministerio de Ciencia y Tecnología MTM2014-52016-C2-02

${ }^{\dagger}$ Corresponding author, eumarti@mat.upv.es 
numerical method many of them become a nonlinear system, $F(x)=0$, where $\mathrm{F}$ is a Fréchet-differentiable operator defined between Banach's spaces.

We deal with the problem of approximating a solution of nonlinear systems. Iterative methods are the common technique used to approximate the solutions.

Many iterative methods can be found in the literature, from the classical Newton or Chebyshev's methods to different variants of these, $[2,3]$. In these studies, authors are interested in giving conditions to obtain a starting point that ensures convergence to a root. Precisely, one can find semilocal and local convergence results in order to estimate the radii of the convergence balls.

The main difference between these types of convergence results is that while in semilocal convergence, $[7,9,4,6,10,8,5]$ one imposes conditions on the starting point $x_{0}$, in the local convergence, $[12,11]$, you impose conditions on the solution. Important results about the convergence domains can be obtained with either local or semilocal techniques.

However, most of the local convergence results are obtained under general conditions that, by using using Taylor's expansions, allow us to find the convergence order but not the radii of the convergence balls; see $[13,14,15]$, among others.

In [5], a semilocal convergence study for a family of third and fourth order methods for nonlinear systems has been performed. A shortcoming of these proofs is that they assume boundedness conditions for high order derivatives, which can be a drawback for solving some practical problems. As can be easily observed in Example 4.1, that is a logarithmic equation, these type of equations appears for obtaining the equilibrium constants in chemical reactions.

In this paper our aim is to obtain a local convergence result for this family of iterative methods for nonlinear equations in Banach spaces, assuming boundedness conditions involving only the first Fréchet derivative, in order to obtain convergence domains. We apply this theory to different examples in order to illustrate the advantages of our results. We include a nonlinear Hammerstein equation that appears in problems of electro-magnetic fluid dynamics, in the kinetic theory of gases and in the reformulation of boundary value problems with a nonlinear boundary condition, $[1,16]$.

Finally, we study the dynamics of the method on complex polynomials of second degree, in order to assess the global convergence properties of the family.

\section{Preliminary results}

Let $X, Y$ be Banach spaces and $F: \Omega \subseteq X \rightarrow Y$ a nonlinear operator in an open convex domain $\Omega_{0} \subseteq \Omega$. We consider the family iterative methods for solving the nonlinear system $F(x)=0$ defined by:

$$
\begin{aligned}
y_{k} & =x_{k}-\theta F^{\prime}\left(x_{k}\right)^{-1} F\left(x_{k}\right) \\
z_{k} & =y_{k}-F^{\prime}\left(x_{k}\right)^{-1} F\left(y_{k}\right) \\
x_{k+1} & =z_{k}-F^{\prime}\left(x_{k}\right)^{-1} F\left(z_{k}\right) .
\end{aligned}
$$


with $\theta \in \mathbb{R} \backslash\{0\}$. In [5], the semilocal convergence analysis of the above mentioned method was made under the following assumptions:

$F$ is twice Fréchet differentiable, $x_{0} \in \Omega_{0}$ is such that $\Gamma_{0}=F^{\prime}\left(x_{0}\right)^{-1}$ exists and the following conditions are verified:

$$
\begin{aligned}
& \left\|\Gamma_{0}\right\| \leq \beta \\
& \left\|\Gamma_{0} F\left(x_{0}\right)\right\| \leq \eta \\
& \left\|F^{\prime \prime}(x)\right\| \leq M, \quad x \in \Omega_{0} \\
& \left\|F^{\prime \prime}(x)-F^{\prime \prime}(y)\right\| \leq K\|x-y\|, \quad x, y \in \Omega_{0} .
\end{aligned}
$$

These conditions involve the existence and boundedness of the Fréchet derivatives $F^{(i)}$ of order $i=1,2,3$, which makes the result very restrictive and difficult to apply to some problems. Our aim is to give a local convergence result for this iterative method relaxing the assumptions of the semilocal convergence case.

From now on, we denote by $B(v, \rho), \bar{B}(v, \rho)$, the open, respectively closed, ball in $X$ with center $v$ and radius $\rho>0$.

\section{Local convergence analysis}

In the local convergence analysis of an iterative method, you impose conditions on the values of $F$ and its derivatives at the solution and obtain a ball centered in the solution such that each point in it can be taken as an starting point for the iterative method. Our aim is to increase the radius of this ball.

In a local convergence study, the conditions are usually expressed in the following way:

$F: \Omega_{0} \subseteq X \rightarrow Y$ is a Fréchet differentiable operator. argyros2015study argyros2015localhl $L_{0}>0, L>0$ and $\theta \in(-\infty, \infty)-\{0\}$ are real numbers verifying that there exists $x^{*} \in \Omega_{0}$ such that, $F\left(x^{*}\right)=0$, and $F^{\prime}\left(x^{*}\right)^{-1} \in L(Y, X)$ and for all $x, y \in \Omega_{0}$ the following holds:

$$
\begin{gathered}
\left\|F^{\prime}\left(x^{*}\right)^{-1}\left(F^{\prime}(x)-F^{\prime}\left(x^{*}\right)\right)\right\| \leq L_{0}\left\|x-x^{*}\right\| \\
\left\|F^{\prime}\left(x^{*}\right)^{-1}\left(F^{\prime}(x)-F^{\prime}(y)\right)\right\| \leq L\|x-y\| .
\end{gathered}
$$

Always, a third assumption is made, as can be observed in different papers, see [21, 22], that can be written as follows:

$$
\left\|F^{\prime}\left(x^{*}\right)^{-1} F^{\prime}(x)\right\| \leq M
$$

for some $M>0$.

Considering the remark made in [21], we can drop this condition. Moreover, a difference from this work and as far as we know, this is the first time that the whole process for establishing local convergence is develop without using constant $M$ imposed in (4). This fact allows us to improve the value of the radius of the convergence ball. For this purpose, we prove the following result: 
Lemma 3.1 If operator $F$ verifies all conditions assumed in (3) then, the following bounds also hold for all $x \in \Omega_{0}$ and $t \in[0,1]$ :

$$
\begin{aligned}
\left\|F^{\prime}\left(x^{*}\right)^{-1} F^{\prime}(x)\right\| & \leq 1+L_{0}\left\|x-x^{*}\right\| \\
\| F^{\prime}\left(x^{*}\right)^{-1}\left(F ^ { \prime } \left(x^{*}+t\left(x-x^{*}\right) \|\right.\right. & \leq 1+L_{0}\left\|x-x^{*}\right\| \\
\left\|F^{\prime}\left(x^{*}\right)^{-1} F(x)\right\| & \leq\left(1+L_{0}\left\|x-x^{*}\right\|\right)\left\|x-x^{*}\right\|
\end{aligned}
$$

Proof: By using the first condition of (3) we have:

$$
\begin{aligned}
\left\|F^{\prime}\left(x^{*}\right)^{-1} F^{\prime}(x)\right\| & \left.=\| F^{\prime}\left(x^{*}\right)^{-1}\left(F^{\prime}(x)-F^{\prime}\left(x^{*}\right)\right)+I\right)\|\leq 1+\| F^{\prime}\left(x^{*}\right)^{-1}\left(F^{\prime}(x)-F^{\prime}\left(x^{*}\right)\right) \| \\
& \leq 1+L_{0}\left\|x-x^{*}\right\|,
\end{aligned}
$$

and then it follows that:

$$
\left\|F^{\prime}\left(x^{*}\right)^{-1}\left(F^{\prime}\left(x^{*}+t\left(x-x^{*}\right)\right)\right)\right\| \leq 1+L_{0} t\left\|x-x^{*}\right\| \leq 1+L_{0}\left\|x-x^{*}\right\|,
$$

being $0<t<1$.

Finally, we use the mean value theorem, so

$$
\begin{aligned}
\left\|F^{\prime}\left(x^{*}\right)^{-1} F(x)\right\| & =\left\|F^{\prime}\left(x^{*}\right)^{-1}\left(F(x)-F\left(x^{*}\right)\right)\right\| \leq\left\|F^{\prime}\left(x^{*}\right)^{-1} F^{\prime}\left(x^{*}+t\left(x-x^{*}\right)\right)\left(x-x^{*}\right)\right\| \\
& \leq\left(\left\|1+L_{0}\right\| x-x^{*} \|\right)\left\|x-x^{*}\right\| .
\end{aligned}
$$

Now we analyse the family of iterative methods, (1), we look for a ball centred at the solution so that any point of this ball can be taking as starting point for the iterative method and the sequence generates remains in this ball and converges to the solution, specifically we want determine de radius of this ball.

So if we denote $x_{0} \in \Omega_{0}$ the starting point and use the assumption (3) we establish by Banach's lemma the first restriction for the domain of convergence:

$$
\left\|F^{\prime}\left(x^{*}\right)^{-1}\left(F^{\prime}\left(x_{0}\right)-F^{\prime}\left(x^{*}\right)\right)\right\| \leq L_{0}\left\|x_{0}-x^{*}\right\|,
$$

so, we impose that $\left\|x_{0}-x^{*}\right\|<\frac{1}{L_{0}}$, as a result by Banach's Lemma on invertible operators, $F^{\prime}\left(x_{0}\right)^{-1}$ exists in $L(Y, X)$ and verifies:

$$
\left\|F^{\prime}\left(x_{0}\right)^{-1} F^{\prime}\left(x^{*}\right)\right\| \leq \frac{1}{1-L_{0}\left\|x_{0}-x^{*}\right\|} .
$$

Therefore, the method is well defined and now we look for bounding the iterates:

$$
\begin{aligned}
y_{0}-x^{*} & =x_{0}-x^{*}-\theta F^{\prime}\left(x_{0}\right)^{-1} F\left(x_{0}\right) \\
& =x_{0}-x^{*}-F^{\prime}\left(x_{0}\right)^{-1} F\left(x_{0}\right)+(1-\theta) F^{\prime}\left(x_{0}\right)^{-1} F\left(x_{0}\right) \\
& =-F^{\prime}\left(x_{0}\right)^{-1}\left(F\left(x_{0}\right)-F^{\prime}\left(x_{0}\right)\left(x_{0}-x^{*}\right)\right)+(1-\theta) F^{\prime}\left(x_{0}\right)^{-1} F\left(x_{0}\right) \\
& =-F^{\prime}\left(x_{0}\right)^{-1} F^{\prime}\left(x^{*}\right) \int_{0}^{1} F^{\prime}\left(x^{*}\right)^{-1}\left[F^{\prime}\left(x^{*}+t\left(x_{0}-x^{*}\right)\right)-F^{\prime}\left(x_{0}\right)\right]\left(x_{0}-x^{*}\right) d t \\
& +(1-\theta) F^{\prime}\left(x_{0}\right)^{-1} F^{\prime}\left(x^{*}\right) \int_{0}^{1} F^{\prime}\left(x^{*}\right)^{-1} F^{\prime}\left(x^{*}+t\left(x_{0}-x^{*}\right)\right)\left(x_{0}-x^{*}\right) d t
\end{aligned}
$$


By taking norms and using (3) and (3.1), we get:

$$
\begin{aligned}
\left\|y_{0}-x^{*}\right\| & \leq\left\|F^{\prime}\left(x_{0}\right)^{-1} F^{\prime}\left(x^{*}\right)\right\|\left\|\int_{0}^{1} F^{\prime}\left(x^{*}\right)^{-1}\left[F^{\prime}\left(x^{*}+t\left(x_{0}-x^{*}\right)\right)-F^{\prime}\left(x_{0}\right)\right]\left(x_{0}-x^{*}\right) d t\right\| \\
& +|1-\theta|\left\|F^{\prime}\left(x_{0}\right)^{-1} F^{\prime}\left(x^{*}\right)\right\|\left\|\int_{0}^{1} F^{\prime}\left(x^{*}\right)^{-1} F^{\prime}\left(x^{*}+t\left(x_{0}-x^{*}\right)\right)\left(x_{0}-x^{*}\right) d t\right\| \\
& \leq \frac{1}{1-L_{0}\left\|x_{0}-x^{*}\right\|}\left[\frac{L}{2}\left\|x_{0}-x^{*}\right\|+|1-\theta|\left(1+L_{0}\left\|x_{0}-x^{*}\right\|\right)\right]\left\|x_{0}-x^{*}\right\| \\
& \leq g_{1}\left(\left\|x_{0}-x^{*}\right\|\right)\left\|x_{0}-x^{*}\right\|,
\end{aligned}
$$

where we have used function $g_{1}(t)$ defined in $\left[0,1 / L_{0}\right]$ as follows:

$$
g_{1}(t)=\frac{1}{1-L_{0} t}\left(\frac{L}{2} t+|1-\theta|\left(1+L_{0} t\right)\right) .
$$

Now, in order to analyze function g1 we define a new function $h_{1}(t)=g_{1}(t)-1$ resulting that $h_{1}(0)=|1-\theta|-1<0$ if $\left.\theta \in\right] 0,2\left[\right.$ and $h_{1}\left(1 / L_{0}\right) \rightarrow+\infty$, so by Bolzano's theorem, we take $r_{1}$ the smallest root of $h_{1}(t)$ in $] 0,1 / L_{0}$ [ and then we have that:

$$
0 \leq g_{1}(t) \leq 1, \forall t \in\left[0, r_{1}\right]
$$

So, if we come back to (7), we have that:

$$
\left\|y_{0}-x^{*}\right\| \leq g_{1}\left(\left\|x_{0}-x^{*}\right\|\right)\left\|x_{0}-x^{*}\right\| \leq\left\|x_{0}-x^{*}\right\| .
$$

Then, we study bounds for the second step in the first iteration of (1). By using (3), (3.1) and (7) we have:

$$
\begin{aligned}
\left\|z_{0}-x^{*}\right\| & \leq\left\|y_{0}-x^{*}\right\|+\left\|F^{\prime}\left(x_{0}\right)^{-1} F\left(y_{0}\right)\right\| \\
& \leq\left\|y_{0}-x^{*}\right\|+\left\|F^{\prime}\left(x_{0}\right)^{-1} F^{\prime}\left(x^{*}\right)\right\|\left\|F^{\prime}\left(x^{*}\right)^{-1} F\left(y_{0}\right)\right\|, \\
& \leq\left(1+\frac{1+L_{0}\left\|y_{0}-x^{*}\right\|}{1-L_{0}\left\|x_{0}-x^{*}\right\|}\right)\left\|y_{0}-x^{*}\right\| \\
& \leq\left(1+\frac{\left.1+L_{0} g_{1}\left(\left\|x_{0}-x^{*}\right\|\right)\left\|x_{0}-x^{*}\right\|\right)}{1-L_{0}\left\|x_{0}-x^{*}\right\|}\right) g_{1}\left(\left\|x_{0}-x^{*}\right\|\right)\left\|x_{0}-x^{*}\right\| \\
& =g_{2}\left(\left\|x_{0}-x^{*}\right\|\right)\left\|x_{0}-x^{*}\right\|,
\end{aligned}
$$

where we have used the function $g_{2}(t)$ defined in $\left[0, r_{1}\right]$ as follows:

$$
g_{2}(t)=\left(1+\frac{1+L_{0} t g_{1}(t)}{1-L_{0} t}\right) g_{1}(t)
$$

Now, we consider function $h_{2}(t)=g_{2}(t)-1$ resulting that $h_{2}(0)=2|1-\theta|-1<0$ if $\theta \in] \frac{1}{2}, \frac{3}{2}\left[\right.$ and $h_{2}\left(r_{1}\right)=\frac{2}{1-L_{0} r_{1}}-1>0$, so by Bolzano's theorem we take $r_{2}$ the smallest root of $h_{2}(t)$ in ]0, $r_{1}[$ and thus we have:

$$
0 \leq g_{2}(t) \leq 1, \quad \forall t \in\left[0, r_{2}\right]
$$


So, coming back to (8), we have:

$$
\left\|z_{0}-x^{*}\right\| \leq g_{2}\left(\left\|x_{0}-x^{*}\right\|\right)\left\|x_{0}-x^{*}\right\| \leq\left\|x_{0}-x^{*}\right\| .
$$

Finally, we analyze the last step:

$$
\begin{aligned}
\left\|x_{1}-x^{*}\right\| & \leq\left\|z_{0}-x^{*}\right\|+\left\|F^{\prime}\left(x_{0}\right)^{-1} F\left(z_{0}\right)\right\| \\
& \leq\left\|z_{0}-x^{*}\right\|+\left\|F^{\prime}\left(x_{0}\right)^{-1} F^{\prime}\left(x^{*}\right)\right\|\left\|F^{\prime}\left(x^{*}\right)^{-1} F\left(z_{0}\right)\right\|, \\
& \leq\left(1+\frac{1+L_{0}\left\|z_{0}-x^{*}\right\|}{1-L_{0}\left\|x_{0}-x^{*}\right\|}\right)\left\|z_{0}-x^{*}\right\| \\
& \leq\left(1+\frac{1+L_{0} g_{2}\left(\left\|x_{0}-x^{*}\right\|\right)\left\|x_{0}-x^{*}\right\|}{1-L_{0}\left\|x_{0}-x^{*}\right\|}\right) g_{2}\left(\left\|x_{0}-x^{*}\right\|\right)\left\|x_{0}-x^{*}\right\| \\
& =g_{3}\left(\left\|x_{0}-x^{*}\right\|\right)\left\|x_{0}-x^{*}\right\|,
\end{aligned}
$$

where we have used the function $g_{3}(t)$ defined in $\left[0, r_{2}\right]$ as follows:

$$
g_{3}(t)=\left(1+\frac{1+L_{0} t g_{2}(t)}{1-L_{0} t}\right) g_{2}(t)
$$

Now, we consider function $h_{3}(t)=g_{3}(t)-1$ resulting that $h_{3}(0)=2 g_{2}(0)-1<$ $4|1-\theta|-1<0$ if $\theta \in] \frac{3}{4}, \frac{5}{4}$ [ and $h_{3}\left(r_{2}\right)=\frac{2}{1-L_{0} r_{2}}-1>0$ so by Bolzano's theorem we take $r_{3}$ the smallest root of $h_{3}(t)$ in $] 0, r_{2}$ [ and so we have:

$$
0 \leq g_{3}(t) \leq 1, \quad \forall t \in\left[0, r_{3}\right]
$$

So, if we come back to (9), we have:

$$
\left\|x_{1}-x^{*}\right\| \leq g_{3}\left(\left\|x_{0}-x^{*}\right\|\right)\left\|x_{0}-x^{*}\right\| \leq\left\|x_{0}-x^{*}\right\| .
$$

We can conclude that for values of $\theta \in] \frac{3}{4}, \frac{5}{4}$ [ we have $0<r_{3}<r_{2}<r_{1}<1 / L_{0}$ so that, by taking

$$
0<r \leq r_{3}: B\left(x^{*}, r\right) \subseteq \Omega_{0}
$$

and starting from any $x_{0} \in B\left(x^{*}, r\right)$ the following relations hold:

$$
\begin{aligned}
\left\|y_{0}-x^{*}\right\| & \leq g_{1}\left(\left\|x_{0}-x^{*}\right\|\right)\left\|x_{0}-x^{*}\right\| \leq\left\|x_{0}-x^{*}\right\|<r, \\
\left\|z_{0}-x^{*}\right\| & \leq g_{2}\left(\left\|x_{0}-x^{*}\right\|\right)\left\|x_{0}-x^{*}\right\| \leq\left\|x_{0}-x^{*}\right\|<r, \\
\left\|x_{1}-x^{*}\right\| & \leq g_{3}\left(\left\|x_{0}-x^{*}\right\|\right)\left\|x_{0}-x^{*}\right\| \leq\left\|x_{0}-x^{*}\right\|<r
\end{aligned}
$$

and thus, the iterates $y_{0}, z_{0}$ and $x_{1}$ remain in $B\left(x^{*}, r\right)$.

The following theorem describes the local convergence analysis of the family of iterative methods (1) using the definitions of the above functions and parameters. 
Theorem 3.1 Let $F: \Omega_{0} \subseteq X \rightarrow Y$ be a Fréchet differentiable operator. Suppose that $L_{0}>0, L>0$ and $\left.\theta \in\right] \frac{3}{4}, \frac{5}{4}$ [ are real numbers such that there exist $x^{*} \in \Omega_{0}$ verifying (3), and let $r$ defined by (10). Then, the sequence $\left\{x_{k}\right\}$ obtained by (1) is well defined for $x_{0} \in B\left(x^{*}, r\right)$, remains in $B\left(x^{*}, r\right)$ for each $k=0,1,2, \ldots$ and converges to $x^{*}$.

Furthermore, if there exists $R \in\left[r, \frac{2}{L_{0}}\right)$ such that $\bar{B}\left(x^{*}, R\right) \subseteq \Omega_{0}$, then the limit point $x^{*}$ is the only solution of the equation $F(x)=0$ in $\bar{B}\left(x^{*}, R\right)$.

Proof: Obviously the whole process we have presented starting by $x_{0}$ obtainig $x_{1}$ can be exactly deduced starting from $x_{k}$ obtaining $x_{k+1}$, just by substituting $x_{0}, y_{0}, z_{0}$ and $x_{1}$ by $x_{k}, y_{k}, z_{k}$, and $x_{k+1}$ in the preceding study, we obtain that all the iterates remain in $B\left(x^{*}, r\right)$, verifying for each $k=0,1,2, \ldots$, the following inequalities:

$$
\begin{aligned}
\left\|y_{k}-x^{*}\right\| & \leq g_{1}\left(\left\|x_{k}-x^{*}\right\|\right)\left\|x_{k}-x^{*}\right\| \leq\left\|x_{k}-x^{*}\right\|<r, \\
\left\|z_{k}-x^{*}\right\| & \leq g_{2}\left(\left\|x_{k}-x^{*}\right\|\right)\left\|x_{k}-x^{*}\right\| \leq\left\|x_{k}-x^{*}\right\|<r, \\
\left\|x_{k+1}-x^{*}\right\| & \leq g_{3}\left(\left\|x_{k}-x^{*}\right\|\right)\left\|x_{k}-x^{*}\right\| \leq\left\|x_{k}-x^{*}\right\|<r
\end{aligned}
$$

Is easy to obtain that function $g_{3}$ is increasing in its domain, so we have:

$$
\begin{aligned}
\left\|x_{k+1}-x^{*}\right\| & \leq g_{3}(r)\left\|x_{k}-x^{*}\right\| \leq g_{3}(r) g_{3}\left(\left\|x_{k-1}-x^{*}\right\|\right)\left\|x_{k-1}-x^{*}\right\| \\
& \leq g_{3}(r)^{2} g_{3}\left(\left\|x_{k-2}-x^{*}\right\|\right)\left\|x_{k-2}-x^{*}\right\| \leq \ldots \leq g_{3}(r)^{k+1}\left\|x_{0}-x^{*}\right\| .
\end{aligned}
$$

Then, by taking limits in the last expression and using that $\lim _{k \rightarrow \infty} g_{3}(r)^{k+1}=0$, we have $\lim _{k \rightarrow \infty} x_{k}=x^{*}$, and so, the method converges to the solution.

In order to prove the uniqueness part, let $y^{*} \in B\left(x^{*}, R\right), y^{*} \neq x^{*}$ with $F\left(y^{*}\right)=0$.

Let us consider the integral operator $T=\int_{0}^{1} F^{\prime}\left(y^{*}+t\left(x^{*}-y^{*}\right)\right) d t$. Then by using (3), we have

$$
\left\|F^{\prime}\left(x^{*}\right)^{-1}\left(T-F^{\prime}\left(x^{*}\right)\right)\right\| \leq \int_{0}^{1} L_{0}\left\|y^{*}+t\left(x^{*}-y^{*}\right)-x^{*}\right\| d t \leq \frac{L_{0}}{2}\left\|x^{*}-y^{*}\right\|=\frac{L_{0}}{2} R<1,
$$

therefore by Banach's Lemma, $T^{-1}$ exists. Then, from the identity

$$
0=F\left(x^{*}\right)-F\left(y^{*}\right)=T\left(x^{*}-y^{*}\right)
$$

we obtain $x^{*}=y^{*}$.

\section{Numerical examples}

In this section the convergence ball for approximating solutions of some nonlinear equations by using methods of the family given by (1) is obtained under weaker hypotheses than before. While the semilocal study presented in [5] involves boundedness conditions 
for high order derivatives, here we only impose the boundedness condition on the first Fréchet derivative (compare conditions (2) with (3)). Most of the examples have been taken from the literature in order to compare the obtained results.

Example 4.1 Let function $f$ defined on $D=\left[-\frac{1}{2}, \frac{5}{2}\right]$ by

$$
f(x)= \begin{cases}x^{3} \ln x^{2}+x^{5}-x^{4}, & x \neq 0 \\ 0, & x=0\end{cases}
$$

First we consider a logarithmic equation that is the typical example in studies of local convergence. The successive derivatives are:

$$
\begin{aligned}
f^{\prime}(x) & =3 x^{2} \ln x^{2}+5 x^{4}-4 x^{3}+2 x^{2} \\
f^{\prime \prime}(x) & =6 x^{2} \ln x^{2}+20 x^{3}-12 x^{3}+10 x \\
f^{\prime \prime \prime}(x) & =6 \ln x^{2}+60 x^{2}-24 x+22 .
\end{aligned}
$$

It can be easily observed that $f^{\prime \prime \prime}$ is unbounded on $D$. So, the results of semilocal convergence involving (2) cannot be applied. However, by applying theorem 3.1 with $x^{*}=1$, we have $L_{0}=L=96.6628$. Taking $\theta=1$, we get:

$$
r_{3}=0.002611<r_{2}=0.004206<r_{1}=0.006897 \text {. }
$$

Example 4.2 We study now a nonlinear integral equation of Hammerstein type. These equations have many applications in chemistry and appear in problems of electro-magnetic fluid dynamics, in kinetic theory of gases, and in the reformulation of boundary value problems, [1],[16]. This equation is of the form:

$$
x(s)=u(s)+\int_{a}^{b} G(s, t) H(x(t)) d t, \quad a \leq s \leq b,
$$

for $x(s), u(s) \in C[a, b]$ with $-\infty<a<b<\infty . G$ is the Green function and $H$ is a polynomial function.

The usual technique to solve these kind of equations consists in expressing it as a nonlinear operator in a Banach space, that is:

$$
F(x)=0,
$$

where $F: \Omega \subseteq C[a, b] \rightarrow C[a, b]$ with $\Omega$ a non-empty open convex subset,

$$
[F(x)](s)=x(s)-u(s)-\int_{a}^{b} G(s, t) H(x(t)) d t,
$$

considering the uniform norm $\|\nu\|=\max _{s \in[a, b]}|\nu(s)|$. 
Observe that in most cases boundedness conditions like (2) cannot be satisfied since $\left\|F^{\prime \prime}(x)\right\|$ or $\left\|F^{\prime \prime \prime}(x)\right\|$ can be unbounded in a general domain. Thus, an alternative is looking a domain that contains the solution. But it is more convenient using the local convergence results obtained in our study in order to give the radius of a convergence ball.

We apply our theoretical study presented in theorem 3.1 to the particular Hammerstein equation given by:

$$
F(x(s))=x(s)-5 \int_{0}^{1} s t x(t)^{3} d t
$$

with $x(s)$ in $\mathcal{C}[0,1]$. The derivative can be written by:

$$
F^{\prime}(x(s)) v(s)=v(s)-15 \int_{0}^{1} s t x(t)^{2} v(s) d t,
$$

One solution of this problem is the null function so it is easy to find different values for Lipschitz constans $L_{0}=7.5$ and $L=15$. By choosing the iterative method from (1) corresponding to $\theta=1$, the following results are obtained:

$$
r_{3}=0.021860<r_{2}=0.037592<r_{1}=0.066667 \text {. }
$$

Example 4.3 Let $X=Y=\mathbb{R}$. Define $F$ on $D=[1,3]$ by

$$
F(x)=\frac{2}{3} x^{\frac{3}{2}}-x
$$

Then, $x^{*}=\frac{9}{4}, F^{\prime}\left(x^{*}\right)^{-1}=2, L_{0}=L=1$.

Choosing

$$
\theta=0.9870,
$$

we have

$$
r_{3}=0.234583<r_{2}=0.390015<r_{1}=0.652346 \text {. }
$$

Example 4.4 Let $X=Y=\mathbb{R}^{3}, D=\bar{U}(0,1)$. Define $F$ on $D$ for $v=(x, y, z)$ by

$$
F(v)=\left(e^{x}-1, \frac{e-1}{2} y^{2}+y, z\right) .
$$

Then the Fréchet derivative is given by

$$
F^{\prime}(v)=\left(\begin{array}{ccc}
e^{x} & 0 & 0 \\
0 & (e-1) y+1 & 0 \\
0 & 0 & 1
\end{array}\right)
$$


Then, $x^{*}=(0,0,0), F^{\prime}\left(x^{*}\right)=F^{\prime}\left(x^{*}\right)^{-1}=\operatorname{diag}\{1,1,1\}, L_{0}=e-1, L=e, M=e$.

Choosing

$$
\theta=1.0125
$$

we have

$$
r_{3}=0.104544<r_{2}=0.181094<r_{1}=0.318661 \text {. }
$$

\subsection{More results}

In Table 4.1 we show for the previous examples the radius of the convergence ball centered at the solution for different values of theta in our family of iterative methods. As you can see the largest radius always corresponds to theta equal one.

\begin{tabular}{|c|c|c|c|c|}
\hline Examples & $\theta=0.85$ & $\theta=0.95$ & $\theta=1$ & $\theta=1.24$ \\
\hline 4.1 & $8.56 \times 10^{-4}$ & $1.942 \times 10^{-3}$ & $2.611 \times 10^{-3}$ & $7.7 \times 10^{-5}$ \\
\hline 4.2 & $7.759 \times 10^{-3}$ & $1.6769 \times 10^{-2}$ & $2.1860 \times 10^{-2}$ & $7.28 \times 10^{-4}$ \\
\hline 4.3 & $8.2782 \times 10^{-2}$ & 0.187715 & 0.252388 & $7.516 \times 10^{-3}$ \\
\hline 4.4 & $3.8647 \times 10^{-2}$ & 0.084765 & 0.111497 & $3.588 \times 10^{-3}$ \\
\hline
\end{tabular}

Table 1: Radii of convergence balls

\subsection{Comparing results}

Now we compare our results with the ones of recent published papers. First, we consider the method defined in [21] that is a family of Chebyshev-Halley-type methods free from second derivative given by:

$$
\begin{aligned}
y_{k} & =x_{k}-F^{\prime}\left(x_{k}\right)^{-1} F\left(x_{k}\right) \\
z_{k} & =x_{k}-\left(1+\left(F\left(x_{k}\right)-2 \alpha F\left(y_{k}\right)\right)^{-1} F\left(y_{k}\right)\right) F^{\prime}\left(x_{k}\right)^{-1} F\left(x_{k}\right) \\
x_{k+1} & =z_{k}-\left(F^{\prime}\left(x_{k}\right)+\bar{F}^{\prime \prime}\left(x_{k}\right)\left(z_{k}-x_{k}\right)\right)^{-1} F\left(z_{k}\right) .
\end{aligned}
$$

where $\bar{F}^{\prime \prime}\left(x_{k}\right)=2 F\left(y_{k}\right) F^{\prime}\left(x_{k}\right)^{2} F\left(x_{k}\right)^{-2}$. The order of convergence of these methods was shown to be at least five and for any value of parameter $\alpha$ and if $\alpha=1$, the order is six.

For this method to work the following condition should be satisfied

$$
0<|\alpha| M L<1
$$

which holds for small values of $\alpha$. For $\alpha=1$, this condition is not satisfied for the considered examples. We have to consider smaller values of $\alpha$ that the authors have considered, we have taken $\alpha=0.00006,0.0075,0.009,0.125$; respectively for examples from 1 to 4 . 
The second method with which we will compare our results defined in [22] has the following iterative function:

$$
\begin{aligned}
y_{k} & =x_{k}-F^{\prime}\left(x_{k}\right)^{-1} F\left(x_{k}\right) \\
u_{k} & =y_{k}+(1-\theta) F^{\prime}\left(x_{k}\right)^{-1} F\left(x_{k}\right) \\
z_{k} & =y_{k}-\gamma A_{\theta, n} F^{\prime}\left(x_{k}\right)^{-1} F\left(x_{k}\right) \\
x_{k+1} & =z_{k}-\alpha B_{\theta, n} F^{\prime}\left(x_{k}\right)^{-1} F\left(z_{k}\right) .
\end{aligned}
$$

where $\alpha, \gamma, \theta \in(\infty, \infty)-\{0\}, H_{\theta, n}=\frac{1}{\theta} F^{\prime}\left(x_{k}\right)^{-1}\left(F^{\prime}\left(u_{k}\right)-F^{\prime}\left(x_{k}\right)\right), A_{\theta, n}=I-\frac{1}{2} H_{\theta, n}(I-$ $\left.\frac{1}{2} H_{\theta, n}\right)$ and $B_{\theta, n}=I-H_{1, n}+H_{\theta, n}^{2}$.

For this method to work, the following conditions should be satisfied.

$$
\begin{aligned}
M|1-\theta| & <1 \\
M|\gamma| & <1 \\
(1+|\alpha| M)|\gamma| M & <1
\end{aligned}
$$

\begin{tabular}{|c|c|c|c|}
\hline Examples & $\theta$ & $\gamma$ & $\alpha$ \\
\hline 4.1 & 1 & 0.005 & 0.008 \\
4.2 & 1 & 0.575 & 0.03 \\
4.3 & 0.987 & $3 / 5$ & 0.001 \\
4.4 & 1.0125 & 0.3 & 0.03 \\
\hline
\end{tabular}

Table 2: Value of the parameters

We have chosen the values of these parameters, see Table 4.2, in such a way that above conditions are satisfied.

From Table 4.2, it can be observed that the present method gives larger radii of convergence than the existing methods. So we conclude that the fact of dropping constant $M$ in the local convergence study has the advantage of obtaining greater convergence balls.

\begin{tabular}{|c|c|c|c|}
\hline Examples & Present method & Method (13) & Method (14) \\
\hline 4.1 & 0.002611 & $4.4 \times 10^{-9}$ & $2.501628 \times 10^{-4}$ \\
\hline 4.2 & 0.021860 & 0.007218 & 0.004999 \\
\hline 4.3 & 0.234583 & 0.105844 & 0.059554 \\
\hline 4.4 & 0.104544 & 0.001728 & 0.027226 \\
\hline
\end{tabular}

Table 3: Comparison of radii of the convergence balls 


\section{Dynamics}

The dynamics of the family of iterative methods 1 has been studied in [5] for systems of nonlinear equations in the real plane. Here we study its dynamical behaviour for complex polynomials of second degree proving scaling and conjugacy results. Similar studies have been performed in $[18,19,20]$ for other families of iterative methods. The dynamics of the relaxed Newton's method has been studied in [17].

The iterates obtained starting from $z_{0} \in \mathbb{C}$ can be denoted by $\left\{z_{0}, R\left(z_{0}\right), R^{2}\left(z_{0}\right), \ldots\right.$, $\left.R^{n}\left(z_{0}\right), \ldots\right\}$, where $R$ is a rational function defined on the Riemann sphere $\hat{\mathbb{C}}$. This set is called the orbit of $z_{0}$.

Let $z \in \hat{\mathbb{C}}$ be a fixed point of the rational function $R$, that is to say $R(z)=z$. The basin of attraction of $z$ consists of the points whose orbit tends to $z$. The behaviour of the orbits near a fixed point $z$ depends on the derivative $R^{\prime}(z)$. If $\left|R^{\prime}(z)\right|<1$, the fixed point $z$ is attracting and if $\left|R^{\prime}(z)\right|>1$, it is repelling. If $R^{\prime}(z)=0$, the fixed point is superattracting.

The set of points $z_{0} \in \hat{\mathbb{C}}$ such that their families $R_{n}\left(z_{0}\right), n \in \mathbb{N}$ are normal in some neighbourhood $U\left(z_{0}\right)$ is the Fatou set, $\mathcal{F}(R)$ and its complement in $\hat{\mathbb{C}}$ is the Julia set $\mathcal{J}(R)$. Roughly speaking, the orbits of the points in $\mathcal{F}(R)$ present a stable behaviour whereas the orbits of the points in $\mathcal{J}(R)$ have chaotic behaviour. In particular, the Fatou set contains the attraction basins of the attracting fixed points whereas the Julia set contains the boundaries of the attraction basins.

Given an analytic function $f(z)$, consider the function associated to a step of the iterative method (1) $M_{f}: \hat{\mathbb{C}} \longrightarrow \hat{\mathbb{C}}$, such that $M_{f}\left(x_{k}\right)=x_{k+1}$. The following scaling result holds for $M_{f}$ :

Theorem 5.1 Let $f$ be an analytic function on $\hat{\mathbb{C}}$, and $A(z)=\alpha z+\beta$, with $\alpha \neq 0$, an affine map. If $g(z)=\lambda(f \circ A)(z), \lambda \neq 0$, then $M_{f}$ is analytically conjugated to $M_{g}$ by $A$, that is, $A \circ M_{g} \circ A^{-1}=M_{f}$.

Any polynomial of second degree is conjugated by an affine transformation to a polynomial of the form $f(z)=z^{2}+c, c \in \mathbb{C}$, so that in order to study the dynamics of $M_{f}$ on quadratic polynomials, it suffices to consider only polynomials of this form.

Then, if $f(z)=z^{2}+c$, with $c \neq 0, M_{f}$ has the form

$$
M_{f}(z)=-\frac{16 z^{4}\left(c+z^{2}\right)\left(c+5 z^{2}\right)+8 z^{2}\left(c+z^{2}\right)^{3} \theta^{2}+\left(c+z^{2}\right)^{4} \theta^{4}}{128 z^{7}} .
$$

The equation $M_{f}(z)=z$ can be written as

$$
\frac{16 z^{4}\left(c+z^{2}\right)\left(c+5 z^{2}\right)+8 z^{2}\left(c+z^{2}\right)^{3} \theta^{2}+\left(c+z^{2}\right)^{4} \theta^{4}}{128 z^{7}}=0,
$$

so that, $M_{f}$ has eight fixed points. Six of them depend on $\theta$ and the two remaining are the roots of $f(z), \pm \sqrt{-c}$, which do not depend on $\theta$. These two fixed points are 


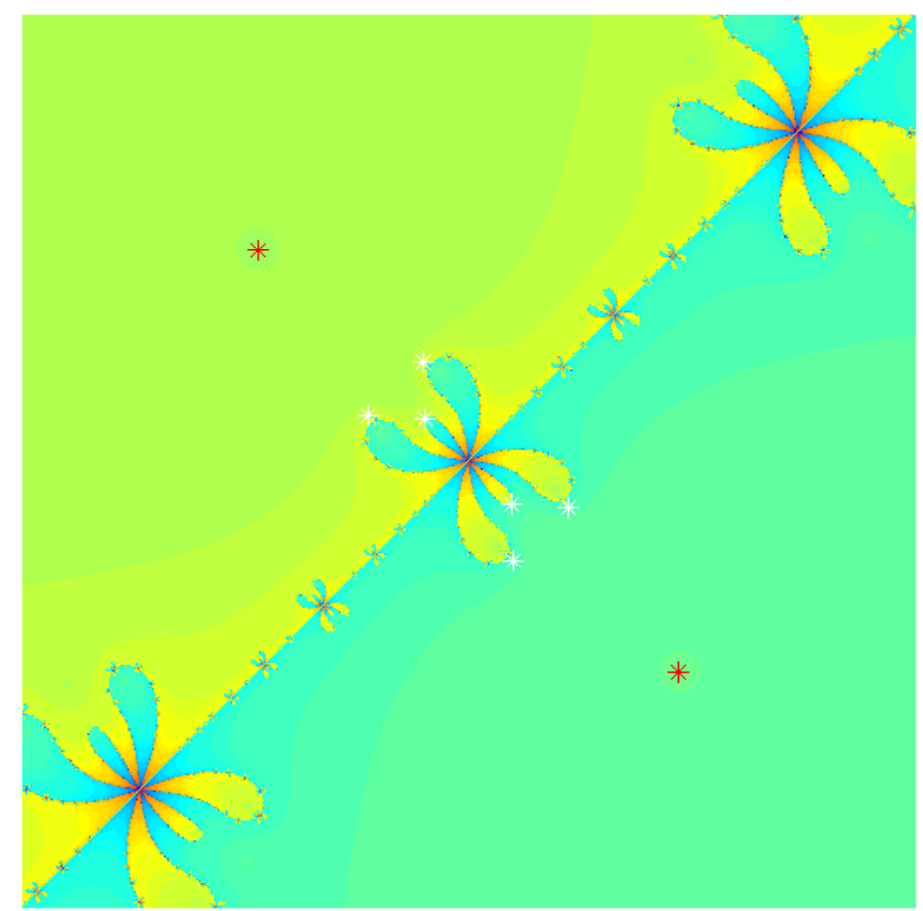

Figure 1: Attraction basins for $c=i$ and $\theta=0.5$.

superattracting, because

$$
M_{f}^{\prime}(z)=\frac{\left(c+z^{2}\right)^{2}\left(48 z^{4}-8 z^{2}\left(-5 c+z^{2}\right) \theta^{2}+\left(7 c-z^{2}\right)\left(c+z^{2}\right) \theta^{4}\right)}{128 z^{8}}
$$

and then, $M_{f}^{\prime}( \pm \sqrt{-c})=0$. The character of the remaining fixed points depends on $\theta$. Figures 1 and 2 show the attraction basins of $M_{f}$ for $f(z)=z^{2}+i$ and values of $\theta$ for which the roots of the polynomial are the only attracting fixed points. The roots, marked in red, are in its attraction basin and the other repelling fixed pints, marked in white, are in the boundary of the basins, in the Julia set.

The dynamical study can be simplified further by using the idea of analytical conjugation. If $\mathrm{B}(\mathrm{z})$ is a Möbius map

$$
B(z)=\frac{\alpha z+\beta}{\gamma z+\delta}, \quad \alpha \delta-\beta \gamma \neq 0
$$

the rational maps $M$ and $N$ are analytically conjugated via $B$ if $N=B M B^{-1}$. Then, $\mathcal{F}(N)=B(\mathcal{F}(M)$ and $\mathcal{J}(N)=B(\mathcal{J}(M)$. 


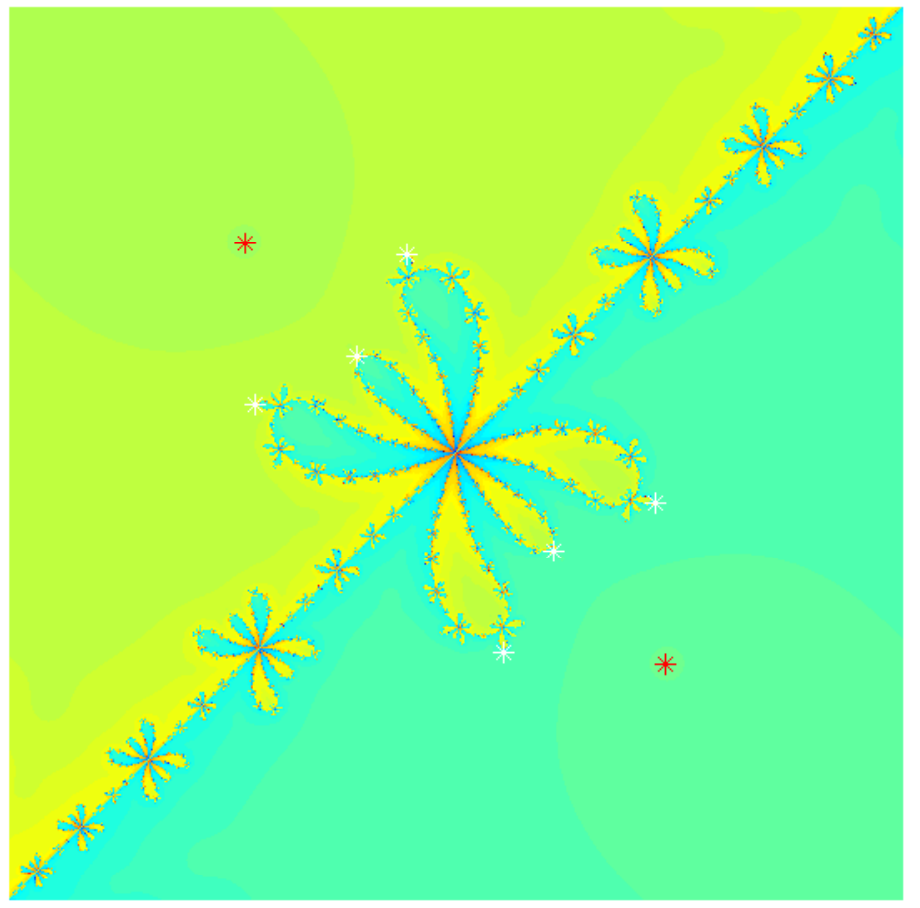

Figure 2: Attraction basins for $c=i$ and $\theta=0.5$. 
Theorem 5.2 Let $f(z)$ be a quadratic polynomial with simple roots. The fixed point operator $M_{f}(z)$ associated to the family of iterative methods (1) verifies:

1. $M_{f}(z)$ is analytically conjugated with

$$
N_{f}(z)=\frac{z^{3}\left(-(1+z)^{4}(2+z)+2(1+z)^{2} \theta^{2}+z \theta^{4}\right)}{-(1+z)^{4}(1+2 z)+2 z^{3}(1+z)^{2} \theta^{2}+z^{4} \theta^{4}} .
$$

2. The Julia set of this operator contains the unit circle.

3. The Fatou set consists of the attraction basins of 0 and infinity. Both are superattracting fixed points.

\section{Proof:}

1. Due to the scaling theorem 5.1, we suppose $f(z)=z^{2}+c$. Then, the Möbius transform

$$
B(z)=\frac{z-\sqrt{-c}}{z+\sqrt{-c}}
$$

has the following properties:

$$
B(\infty)=1, \quad B(\sqrt{-c})=0, \text { and } B(-\sqrt{-c})=\infty .
$$

By conjugating $M_{f}$ with $B$ one gets (19), which does not depend on $c$.

2. It is easy to check that the unit circle $z:|z|=1$ is invariant under $N_{f}$.

3. Expression (19) shows that 0 and $\infty$ are superattracting fixed points of third order.

Figures 3 and 4 depict the Julia sets of $N_{f}$ for $\theta=0.5$ and $\theta=1.5$, respectively. The Julia set of Newton's method for polynomials of second degree reduces to the unit circle. In our case, the set is more involved, but still includes it.

The figures show that most of the points in the complex plane belong to the attraction basin of a root, so that, they are suitable as starting points for the iterations of the considered family of methods, when dealing with quadratic polynomials. This implies a good global behaviour of the family, in comparison with other iterative methods.

\section{References}

[1] I.K. Argyros, S. Hilout, M.A. Tabatabai Mathematical Modelling with Applications in Biosciences and Engineering Nova Publishers, New York, 2011.

[2] Traub, J. F. Iterative Methods for the Solution of Equations Prentice-Hall, Englewood Cliffs, New Jersey (1964). 


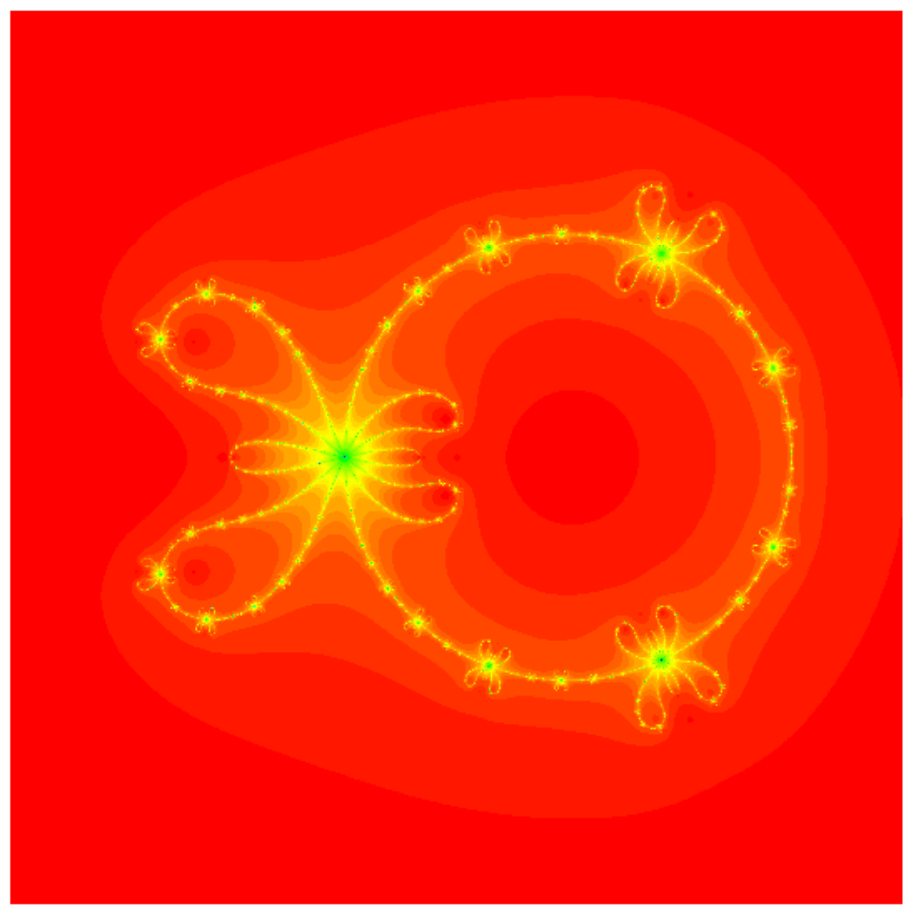

Figure 3: Julia set of $N_{f}$ for $\theta=0.5$

[3] Ostrowski, A. M. Solutions of Equations in Euclidean and Banach Spaces Academic Press, New York (1973).

[4] I.K. Argyros, J.A. Ezquerro, J.M. Gutirrez, M.A. Hernández, S. Hilout, On the semilocal convergence of efficient ChebyshevSecant-type methods J. Comput. Appl. Math. 235 (2011) 3195-3206.

[5] José L.Hueso, Eulalia Martínez Semilocal convergence of a family of iterative methods in Banach spaces Numer Algor (2014) 67 365-384.

[6] Wang, X., Gu, C., Kou, J. Semilocal convergence of a multipoint fourth-order superHalley method in Banach spaces, Numerical Algorithms, 54, (2011), 497-516.

[7] Kou, J., Li, Y., Wang, X., A variant of super Halley method with accelerated fourthorder convergence, Applied Mathematics and Computations, 186, (2007), 535-539.

[8] Zheng, L., Gu, C., Recurrence relations for semilocal convergence of a fifth-order method in Banach spaces, Numerical Algorithms, 59, (2012), 623-638. 


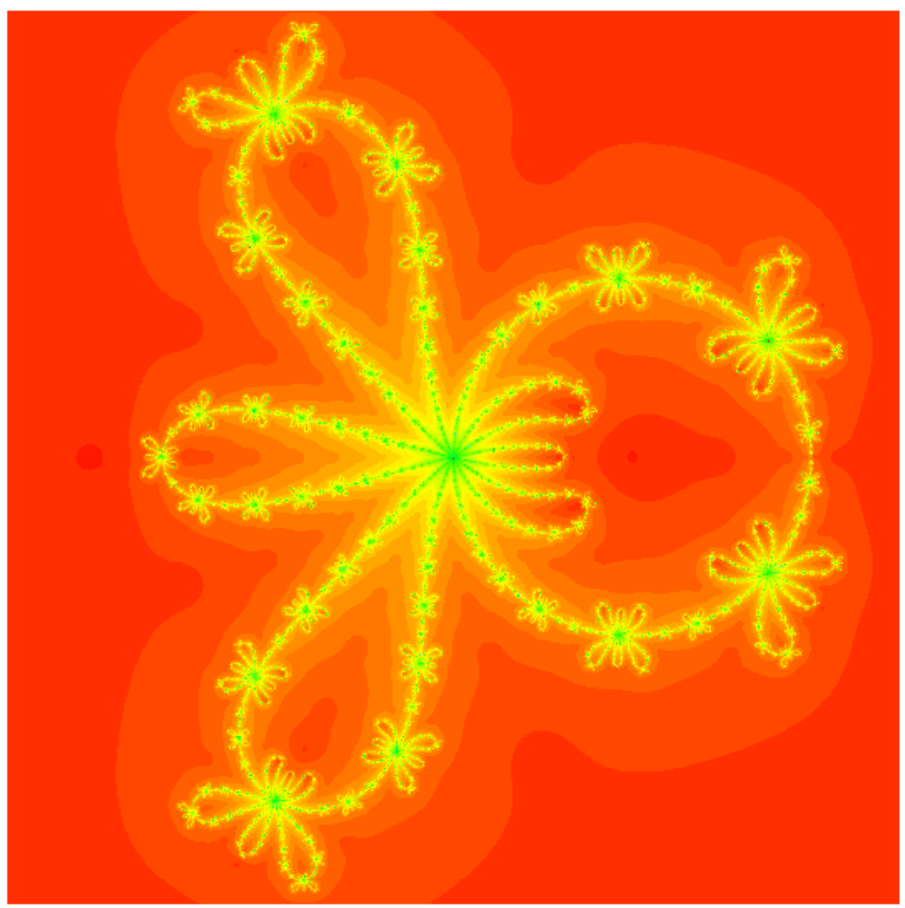

Figure 4: Julia set of $N_{f}$ for $\theta=1.5$

[9] Amat, S., Hernández, M.A., Romero, N., A modified Chebyshevs iterative method with at least sixth order of convergence, Applied Mathematics and Computation, 206, (2008), 164-174.

[10] Wang, X., Kou, J., Gu, C., Semilocal convergence of a sixth-order Jarratt method in Banach spaces, Numerical Algorithms, 57, (2011), 441-456.

[11] A. Cordero, J.A. Ezquerro, M.A. Hernández-Verón, J.R. Torregrosa. On the local convergence of a fifth-order iterative method in Banach spaces, Applied Mathematics and Computation 251 (2015) 396-403.

[12] I.K. Argyros, S. Hilout, On the local convergence of fast two-step Newton-like methods for solving nonlinear equations Journal of Computational and Applied Mathematics 245 (2013) 1-9

[13] S. Weerakoon, T.G.I. Fernando, A variant of Newton's method with accelerated third-order convergence, Applied Mathematics Letters, 13 8, pp. 87-93, (2000). 
[14] X. Feng, Y. He, High order oterative methods without derivatives for solving nonlinear equations Applied Mathematics and Computation, 186 (2007) 1617-1623.

[15] X. Wang, J. Kou, Y. Li, Modified Jarratt method with sixth-order convergence, Applied Mathematics Letters, 22, pp. 1798-1802, (2009).

[16] A.D. Polyanin, A.V. Manzhirov, Handbook of Integral Equations, CRC Press, Boca Raton, 1998.

[17] S. Plaza, Natalia Romero, Attracting cycles for the relaxed Newton's method, Journal of Computational and Applied Mathematics, Volume 235, Issue 10, 15 March 2011, Pages 3238-3244.

[18] Alicia Cordero, Juan R. Torregrosa , Pura Vindel Study of the dynamics of thirdorder iterative methods on quadratic polynomials International Journal of Computer Mathematics. Vol. 89, Iss. 13-14, (2012).

[19] Gerardo Honorato, Sergio Plaza, Natalia Romero, Dynamics of a higher-order family of iterative methods, Journal of Complexity Volume 27 Issue 2, April, (2011) Pages 221-229.

[20] J.M. Gutirrez, M.A. Hernández, N. Romero, Dynamics of a new family of iterative processes for quadratic polynomials, Journal of Computational and Applied Mathematics, Volume 233, Issue 10, 15 March 2010, Pages 2688-2695.

[21] I.K. Argyros, A. A. Magreñan, A study on the local convergence and dynamics of Chebyshev-Halley-type methods free from second derivative, Numerical Algorithms, DOI 10.1007/s11075-015-9981-x.

[22] I.K. Argyros, S. George, Local convergence of modified Halley-like methods with less computation of inversion, Novi Sad J. Math, Draft version, (2015). 